\title{
Thoracic interfascial plane block for multimodal analgesia after breast lumpectomy
}

\section{Yeojung Kim, Chahyun Oh, Sookyoung Youn, Sangwon Yun, Hyunwoo Park, Wonhyung Lee, Yoon-Hee Kim, YoungKwon Ko, and Boohwi Hong}

Received September 11, 2018

Revised October 22, 2018

Accepted November 5, 2018

\section{Corresponding author}

Boohwi Hong, M.D., Ph.D.

Department of Anesthesiology and

Pain Medicine, Chungnam National

University Hospital, 282 Munhwa-ro,

Jung-gu, Daejeon 35015, Korea

Tel: 82-42-280-7844

Fax: 82-42-280-7968

E-mail: koho0127@gmail.com

ORCID

https://orcid.org/0000-0003-2468-9271
Department of Anesthesiology and Pain Medicine, Chungnam National University Hospital, Daejeon, Korea

Background: Thoracic interfascial plane block is useful as a component of multimodal analgesia in patients undergoing mastectomy. However, multimodal analgesia tends not to be provided during lumpectomy as it is one of the less aggressive procedures among breast cancer surgeries. Therefore, we investigated the effects of thoracic interfascial plane block as more effective analgesia after breast lumpectomy.

Methods: Forty six patients (20-80 years old, female) with breast cancer scheduled to undergo lumpectomy were randomly assigned to two groups. Postoperative pain control in the control group consisted only of intravenous patient-controlled analgesia (PCA). In the block group, intravenous PCA was used after serratus intercostal fascial plane block and pecto-intercostal fascial plane block. The primary outcome was the $24 \mathrm{~h}$ cumulative postoperative fentanyl consumption. Pain severity, additional rescue analgesic requirement, side effects, and patient satisfaction were also evaluated.

Results: Postoperative fentanyl consumption in the block group was significantly reduced compared with the control group (median, 88.8 [interquartile range, 48.0, 167.6] vs. $155.2[88.8,249.2], P=0.022)$. The pain score was significantly lower in the block group only in the post-anesthesia care unit ( $2.9 \pm 1.8$ vs. $4.3 \pm 2.3, P=0.022)$. There were no differences in the incidence of postoperative nausea and vomiting and the requirement for additional analgesics between the groups. The satisfaction score was significantly higher in the block group.

Conclusions: Thoracic interfascial plane block after lumpectomy reduces opioid usage and increases patient satisfaction with postoperative pain control. Thoracic interfascial plane block is useful for multimodal analgesia after lumpectomy.

Keywords: Analgesics, opioid; Mastectomy, segmental; Nerve block; Postoperative pain.

\section{INTRODUCTION}

Breast cancer surgery is frequently performed in women and it can result in chronic pain as well as acute postoperative pain [1-4]. Lumpectomy as one of the less aggressive operations among breast cancer surgeries involves resection of some subcutaneous tissues and may include axillary lymph node dissection or sentinel lymph node biopsy [5]. Because the extent of lumpectomy is smaller than that of other breast surgeries, most patients tend not to receive aggressive pain control following surgery. However, postoperative pain after lumpectomy can be severe enough to delay recovery by interfering with sleep and daily activities. Therefore, sufficient pain control is essential even after lumpectomy.

This is an Open Access article distributed under the terms of the Creative Commons Attribution Non-Commercial License (http://creativecommons.org/licenses/by-nc/4.0) which permits unrestricted non-commercial use, distribution, and reproduction in any medium, provided the original work is properly cited. 
Opioid analgesics and nonsteroidal anti-inflammatory drugs, commonly used after surgery, have limitations owing to side effects such as nausea, vomiting [6], and respiratory depression. On the other hand, various thoracic nerve blocks performed for pain control after breast cancer surgery provide superior analgesic effect and reduce postoperative nausea and vomiting as a result of the decreased use of opioid analgesics [7-14]. There have been several reports that thoracic interfascial plane block is useful for multimodal analgesia in patients undergoing mastectomy [15-17]. The simultaneous use of thoracic interfascial plane block and intravenous patient-controlled analgesia (PCA) for multimodal analgia has been shown to be effective in pain control following mastectomy [15-17].

Thoracic interfascial plane block, including pecto-intercostal fascial plane block (PIFB) $[13,18,19]$ and serratus intercostal fascial plane block (SIFB) [20-23], is the peripheral nerve block that targets the intercostal nerves branches distributed in the chest and axilla [11-23]. Although PIFB and SIFB are thought to be relatively easy to perform, there have been no reports of the simultaneous performance of the two blocks.

In this prospective randomized observer-blinded study, we hypothesized that the thoracic interfascial plane block can effectively control pain as part of multimodal analgesia after lumpectomy, reduce side effects, and increase patient satisfaction by reducing consumption of opioids and other additional analgesics.

\section{MATERIALS AND METHODS}

This study was approved by the committee of the Institutional Review Board of our hospital (CNUH 2016-02-018). Female patients (American Society of Anesthesiologists physical status I to III, 20 to 80 years old) with breast cancer undergoing lumpectomy with or without axillary lymph node dissection from April 2016 to October 2016 were assessed for eligibility at our hospital, with all participating patients providing written informed consent. Study exclusion criteria included patient refusal, American Society of Anesthesiologists physical status IV, local anesthetic allergy, pre-existing neurologic impairment in block region, previous history of intravenous PCA side effects, intravenous PCA refusal, and bleeding tendency (coagulopathy, thrombocytopenia, antiplatelet/anticoagulation medication). A total of 46 patients with breast cancer scheduled to undergo lumpectomy were randomly allocated to one of two groups. Postoperative pain control in the control group consisted only of intravenous PCA. In the block group, intravenous PCA was used after the performance of SIFB $(0.375 \%$ ropivacaine $20 \mathrm{ml})$ and PIFB ( $0.375 \%$ ropivacaine $20 \mathrm{ml}$ ) after surgery. Patients were randomized using a computer generated random table uploaded on REDCap software (Vanderbilt University, USA) for management of data.

Intravenous glycopyrrolate $0.2 \mathrm{mg}$ was administered 30 min before surgery. Percutaneous arterial oxygen saturation $\left(\mathrm{SpO}_{2}\right)$, electrocardiogram, noninvasive blood pressure (NIBP), and bispectral index (BIS) were monitored in the operating room. Induction of general anesthesia was performed with propofol 1.5-2 mg/kg and remifentanil $50 \mu \mathrm{g}$, followed by rocuronium $0.6 \mathrm{mg} / \mathrm{kg}$. Anesthesia was maintained with 4-6\% desflurane and 40-50\% oxygen in air, and remifentanil $0.05-0.15 \mu \mathrm{g} / \mathrm{kg} / \mathrm{h}$ continuous infusion. The depth of anesthesia was modulated according to vital signs and BIS. Heart rate (HR) and NIBP were maintained within $20 \%$ of each patient's baseline values. BIS was maintained within 40-60. Intravenous PCA was a concentration of fentanyl $5 \mu \mathrm{g} / \mathrm{ml}$ with $500 \mu \mathrm{g}$ of fentanyl mixed with normal saline. A dose of fentanyl $0.5 \mu \mathrm{g} / \mathrm{kg}$ was administered just prior to anesthesia emergence, with a set dose of fentanyl $20 \mu \mathrm{g}$ at least every 10 $\min$.

SIFB and PIFB were performed after surgery and just before anesthesia emergence. The investigator was seated at the head of the patient, the ultrasound device (Mylab ${ }^{\mathrm{TM}} 25$ Gold, Esaote, Italy) was positioned diagonally to the investigator, and the surgical side of breast was placed at the center.

For the performance of SIFB, the linear probe (LA435: 6-18 $\mathrm{MHz}$, Esaote) was placed below the outer third of the clavicle to detect anatomical landmarks, such as the serratus anterior muscle (SAM), external intercostal muscle (EIM), pectoralis muscles, thoracoacromial artery, and second rib. A 22-gauge Quincke type spinal needle was inserted in a medial to lateral downward direction below the clavicle using the in-plane technique. The needle tip was placed between the SAM and the second rib or EIM $[22,23]$. A test bolus of $1-2 \mathrm{ml}$ of $0.375 \%$ ropivacaine was injected; upon confirmation of diffusion of the test dose between the external intercostal muscles and the SAM, a total of $20 \mathrm{ml}$ of local anesthetic was injected. The needle was then carefully moved in the direction of the third 
and fourth ribs while confirming expansion of the fascial plane.

In the performance of PIFB, a probe was placed parallel to the long axis of the sternum at a distance greater than $2 \mathrm{~cm}$ from the attachment of the second rib and sternum to identify the pectoralis muscles, EIM, and second rib. After a 22-gauge Quincke type spinal needle was inserted in the caudad direction using the in-plane technique, the needle tip was located at the attachment point of the pectoralis muscles and second rib $[13,18,19]$. After confirmation that $1-2 \mathrm{ml}$ of $0.375 \%$ ropivacaine was spread between the EIM and the pectoralis muscles, a total of $20 \mathrm{ml}$ of local anesthetic was injected. The needle was then carefully moved in a caudad direction while confirming expansion of the fascial plane.

Considering the toxic dose of ropivacaine ( $3 \mathrm{mg} / \mathrm{kg}$ ), the total amount of local anesthetic was limited to $30 \mathrm{ml}$ when the patient's weight was less than $50 \mathrm{~kg}$.

To minimize variations in the techniques, the blocks were performed by a single experienced investigator who was then excluded from postoperative outcome evaluation. The corresponding block needle insertion site was covered with a 3 $\times 4 \mathrm{~cm}$ bandage and a compressible breast pad was placed over the surgical site to allow blinding of the postoperative outcome evaluator to group allocation.

All patients were educated before surgery regarding the numeric rating scale (NRS) pain scoring system used to evaluate postoperative pain (0: no pain, 10: worst pain). Additionally, the axillary pain assessment was based on the NRS pain score that was felt when the axillary area was tapped.

In the case of an NRS pain score of 4 or higher, an additional intravenous dose of fentanyl $0.5 \mu \mathrm{g} / \mathrm{kg}$ was administered by intravenous PCA, after which the patient was monitored for adverse effects such as respiratory depression, nausea, and vomiting. In case of difficulties with pain control using intravenous PCA due to opioid induced side effects, intravenous ketorolac $30 \mathrm{mg}$ was administered.

Postoperative fentanyl consumption for the first $24 \mathrm{~h}$ using intravenous PCA was investigated as the primary outcome. The secondary outcomes were postoperative NRS pain score, postoperative nausea and vomiting (PONV), and patient satisfaction (0-10 points) in relation to pain control during the first $24 \mathrm{~h}$ postoperative assessed through questionnaires. The use of additional analgesics was confirmed by review of medical records.

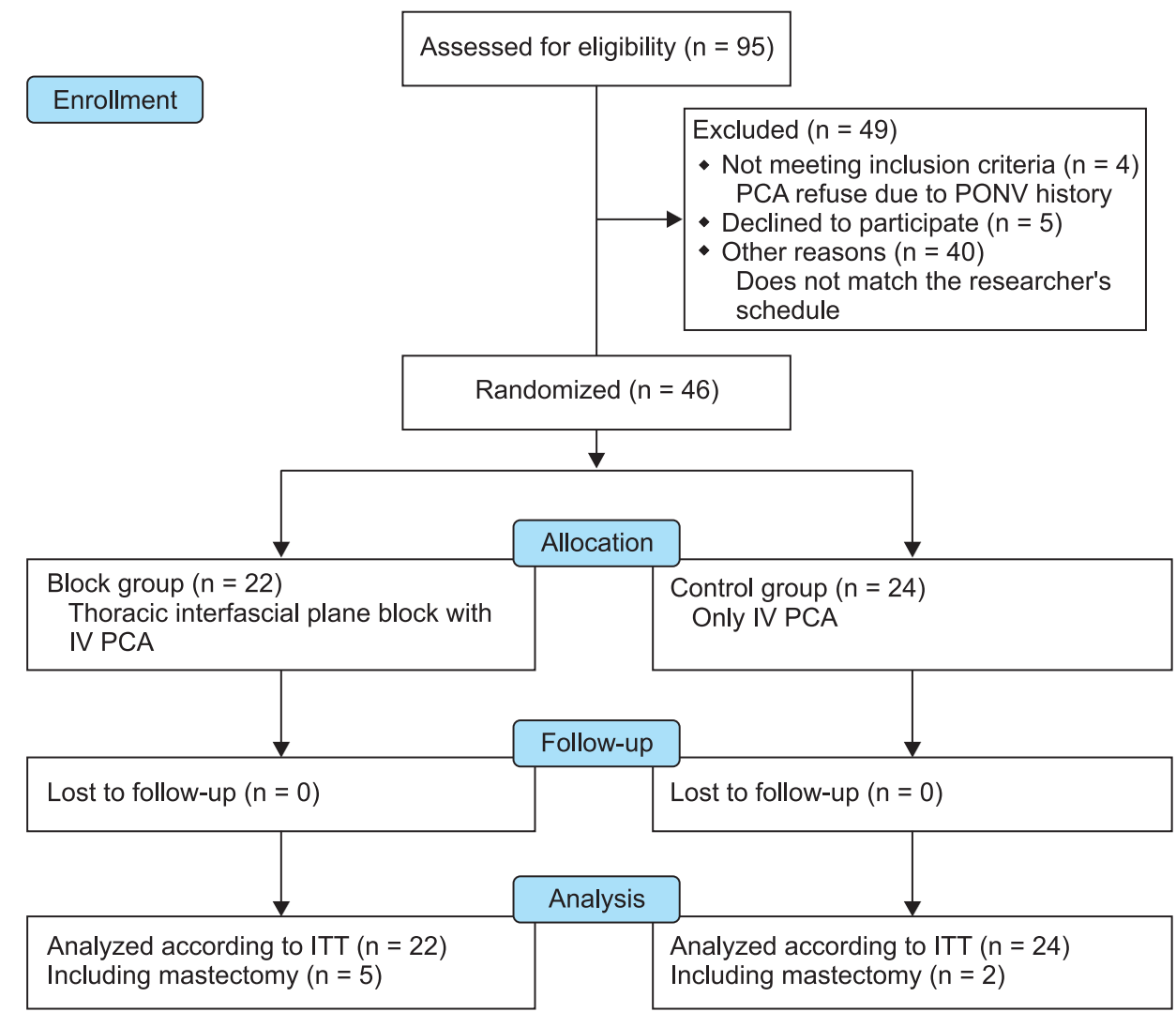

Fig. 1. Consolidated Standards of Reporting Trials (CONSORT) flow diagram of a randomized trial of two groups. PCA: patient-controlled analgesia, PONV: postoperative nausea and vomiting, IV: intravenous, ITT: intention to treat. 
The required sample size was calculated based on a sample of 20 consecutive patients who underwent lumpectomy. The observed postoperative fentanyl consumption for the first $24 \mathrm{~h}$ was $164.4 \pm 95.0$. Assuming a $50 \%$ reduction in opioid consumption and a power of $80 \%$ with a risk of 0.05 for type 1 errors (two tailed, effect size of 0.87 ), the minimum number of patients required in each group was 22 . Allowing for a $10 \%$ dropout rate, we planned to recruit a total of 48 patients.

All analyses were performed according to intention to treat, so patients who were converted to mastectomy were also included. After the exclusion of mastectomy, the primary outcome, fentanyl consumption for the first $24 \mathrm{~h}$, was further analyzed. Normality was tested using the Shapiro-Wilk test. Continuous variables were recorded as mean \pm standard deviation (SD) or median (interquartile range), and differences were analyzed using independent $t$-tests or Mann-Whitney $U$ tests, depending on the results of normality testing. Quantitative variables were reported as number (\%) and compared using $\chi^{2}$ tests or Fisher's exact tests. P values $<0.05$ were considered statistically significant. Statistical analyses were performed using R software version 3.4.2 (R Project for Statistical Computing, Austria). Point estimation and the confidence interval of the Hodges-Lehmann's median were calculated

Table 1. Demographic Data

\begin{tabular}{lcc}
\hline \multicolumn{1}{c}{ Variable } & Block group $(\mathrm{n}=22)$ & Control group $(\mathrm{n}=24)$ \\
\hline Age $(\mathrm{yr})$ & $57.9 \pm 12.0$ & $50.5 \pm 9.2$ \\
Height $(\mathrm{cm})$ & $155.8 \pm 6.9$ & $157.8 \pm 5.1$ \\
Weight $(\mathrm{kg})$ & $57.5 \pm 7.3$ & $60.9 \pm 8.4$ \\
$\mathrm{BMI}\left(\mathrm{kg} / \mathrm{m}^{2}\right)$ & $23.7 \pm 2.9$ & $24.5 \pm 3.6$ \\
Surgeon $(\mathrm{A} / \mathrm{B})$ & $13(59.1) / 9(40.9)$ & $13(54.2) / 11(45.8)$ \\
Operation time $(\mathrm{min})$ & $102.0(870.0,148.0)$ & $103.0(88.0,126.5)$ \\
Anesthesia time $(\mathrm{min})$ & $132.0(125.0,175.0)$ & $125.5(113.0,144.5)$ \\
PACU stay time $(\mathrm{min})$ & $34.0(30.0,40.0)$ & $35.5(30.0,44.0)$ \\
Operation change to & $5(22.7)$ & $2(8.3)$ \\
$\quad$ mastectomy & & \\
Axillary node & $7(31.8)$ & $4(16.7)$ \\
$\quad$ dissection & & \\
Involved breast & & \\
quadrant & & \\
$\quad$ Upper lateral & $16(72.7)$ & $9(37.5)$ \\
$\quad$ Upper medial & $4(18.2)$ & $3(12.5)$ \\
$\quad$ Lower lateral & $5(22.7)$ & $3(12.5)$ \\
$\quad$ Lower medial & $0(0)$ & $593.8 \pm 252.5$ \\
Intraoperative fluid $(\mathrm{ml})$ & $661.4 \pm 230.9$ & $2(8.3)$ \\
Smoker & $1(4.5)$ &
\end{tabular}

Values are presented as mean \pm SD, number (\%), or median (1Q, 3Q). BMI: body mass index, PACU: post-anesthesia care unit. using SAS software (version 9.3 for windows, SAS Institute, USA).

\section{RESULTS}

A total of 95 patients were assessed for eligibility; of these, 49 were excluded. Subsequently, 46 patients were randomized, with 22 assigned to the block group and 24 to the control group. None of the participants were lost to follow-up after exclusion; therefore all patients were analyzed. The Consolidated Standards of Reporting Trials diagram is shown in Fig. 1. The demographic and clinical characteristics of the two groups are shown in Table 1.

Postoperative 24-h fentanyl consumption in the block group was significantly lower than that in the control group. The median postoperative 24 -h fentanyl consumption was $88.8 \mu \mathrm{g}$ (interquartile range [48.0, $167.6 \mu \mathrm{g}]$ ) in the block group, compared with $155.2 \mu \mathrm{g}(88.8,249.2 \mu \mathrm{g})$ in the control group ( $\mathrm{P}=0.022$ ) (Fig. 2). The Hodges-Lehmann median difference was $66.7 \mu \mathrm{g}$ (95\% confidence interval [CI], 7.4-126.0 $\mu \mathrm{g})$ between the groups. The weight-adjusted fentanyl consumption was also significantly lower in the block group (1.7 $[0.9,2.7]$ vs. $2.3[1.5,4.8] \mu \mathrm{g} / \mathrm{kg}, \mathrm{P}=0.039)$, with a HodgesLehmann median difference of 0.9 (95\% CI, 0.05-1.8) $\mu \mathrm{g} /$ $\mathrm{kg}$ between the groups. After excluding the patients who underwent mastectomy, fentanyl consumption for the first 24 $\mathrm{h}$ was also significantly reduced in the block group (91.0 [48.0, 137.5] vs. 140.5 [86.0, 226.0], $\mathrm{P}=0.031)$.

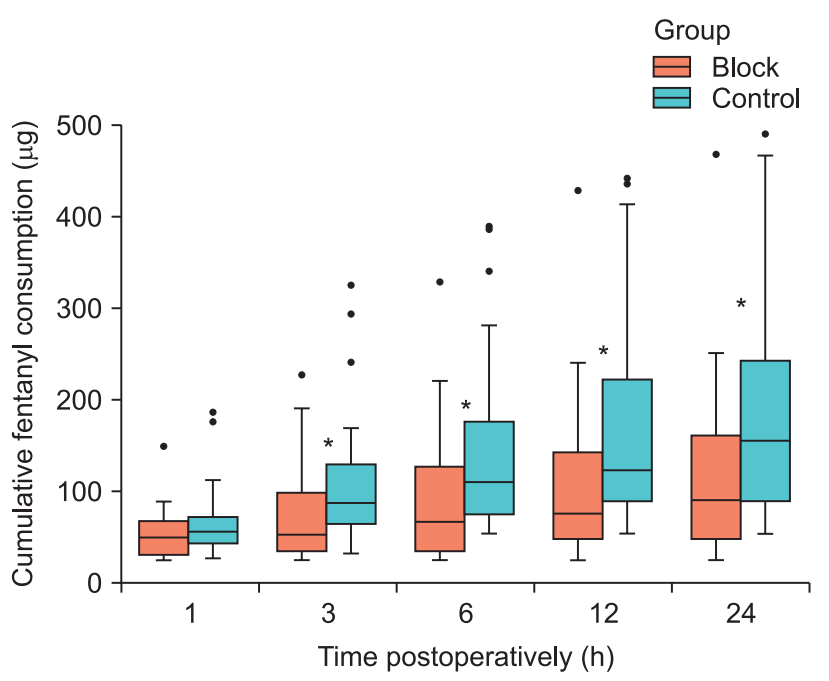

Fig. 2. Postoperative fentanyl consumption for first $24 \mathrm{~h} . * \mathrm{P}<0.05$ between two groups. 
The postoperative NRS pain scores were significantly lower in the block group only in the post-anesthesia care unit (PACU). The NRS pain score in the block group in the PACU was $2.9 \pm 1.8$ (mean $\pm \mathrm{SD}$ ), compared with $4.3 \pm 2.3$ in the control group $(\mathrm{P}=0.022)$. However, there were no differences in the NRS pain score between the block group and the control group after $3 \mathrm{~h}$ postoperatively (Fig. 3). Axillary pain assessed by mild tapping in the PACU was significantly reduced in the block group compared to the control group ( $3.0 \pm 1.9$ vs. $4.5 \pm$ $2.5, \mathrm{P}=0.023)$.

There were no differences between the groups in the rate of PONV and the use of additional analgesics. Satisfaction scores were significantly higher in the block group. The median satisfaction score was 9 [8-10] in the block group, compared with 8 [7-9] in the control group $(\mathrm{P}=0.043)$ (Table 2). There were no complications in either of the groups.

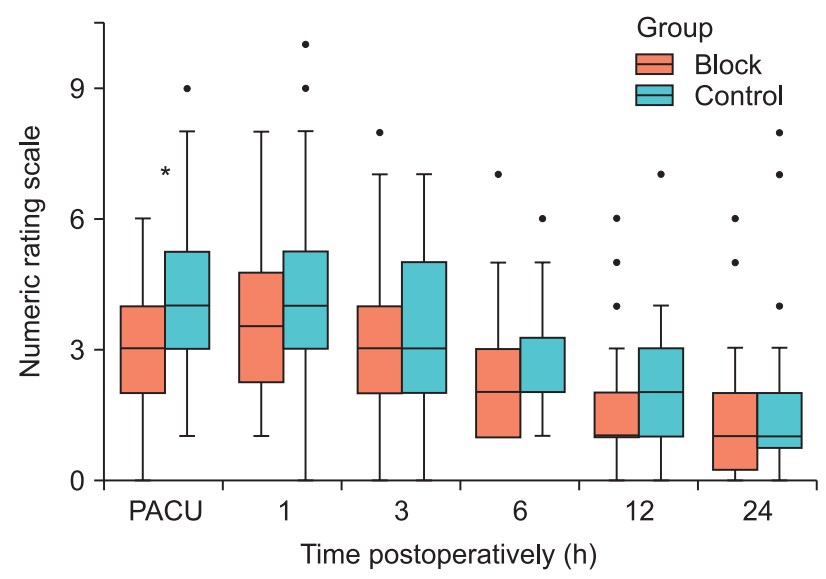

Fig. 3. Postoperative numeric rating scale pain scores. PACU: postanesthesia care unit. $* \mathrm{P}<0.05$ between two groups.

\section{DISCUSSION}

The present study showed that thoracic interfascial plane blocks (PIFB and SIFB) after breast lumpectomy can reduce postoperative opioid consumption, and thoracic interfascial plane blocks with intravenous PCA for multimodal analgesia result in superior patient satisfaction with pain control compared to intravenous PCA alone.

Several methods for multimodal analgesia after breast cancer surgery have been used in clinical practice. For example, various nerve blocks are used. Although the commonly used thoracic epidural nerve block and thoracic paravertebral block provide excellent pain relief, these procedures are difficult to perform, with associated risks involved in changing the patient's posture for the procedure, and potential hazards such as pneumothorax and neurological complications [710]. Therefore, thoracic interfascial plane block has emerged as a pain control method in individuals undergoing breast cancer surgery because it is safe, not difficult to perform, and does not require changing the patient's posture. There have been several reports that thoracic interfascial plane block was effective as multimodal analgesia in breast cancer surgeries such as modified mastectomy and simple mastectomy [15-17]. However, the present study is the first to investigate the analgesic effect of the simultaneous performance of SIFB and PIFB after breast lumpectomy.

The resection area of breast cancer surgery depends on the stage and extent of the cancer. Lumpectomy as a breast cancer surgery is a procedure for wedge resection of subcutaneous breast tissue. It is known that the 2nd through 6th thoracic intercostal nerves and the pectoral nerve, a branch of the brachial plexus, are related to the pain following breast surgery [24,25]. In a lumpectomy, depending on whether

Table 2. Postoperative Outcomes

\begin{tabular}{lccc}
\hline \multicolumn{1}{c}{ Outcomes } & Block group $(\mathrm{n}=22)$ & Control group $(\mathrm{n}=24)$ & P value \\
\hline Axillary pain in PACU & $3.0 \pm 1.9$ & $4.5 \pm 2.5$ & 0.023 \\
PONV grade $(0 / 1 / 2 / 3)$ & & & 0.609 \\
PACU & $20 / 2 / 1 / 1$ & $17 / 4 / 1 / 0$ & 0.408 \\
Ward & $16 / 5 / 1 / 2$ & $13 / 7 / 2 / 0$ & 0.787 \\
Frequency of additional analgesics & $17 / 3 / 2 / 0$ & $17 / 4 / 2 / 1$ & \\
(0/1/2/3) & & & 0.043 \\
Satisfaction score & $9.0(8.0,10.0)$ & $8.0(7.0,9.0)$ & \\
\hline
\end{tabular}

Values are presented as mean \pm SD, number only, or median $(1 \mathrm{Q}, 3 \mathrm{Q})$. Axillary pain in PACU was evaluated mild tapping in axillary area. PONV: postoperative nausea and vomiting, PACU: post-anesthesia care unit. 
surgery is performed lateral or medial to the nipple, lateral or anterior cutaneous branches of the intercostal nerves, respectively, will contribute to the innervation of the surgical area [5].

PIFB and SIFB are nerve blocks targeting the intercostal nerve branches of the medial and lateral breast, respectively [13]. PIFB anesthetizes the anterior cutaneous branches of the intercostal nerve $[5,13]$. SIFB anesthetizes the lateral cutaneous branches and the intercostobrachial nerve of the intercostal nerve $[5,13]$.

PIFB and SIFB as used in this study were theoretically expected to block branches of the intercostal nerve in the chest and significantly reduce pain after lumpectomy $[5,17,26,27]$.

Because breast lumpectomy involves resection of some subcutaneous tissues but does not expose the pectoralis muscles, there was no injection between the pectoralis major and minor for pectoral nerve block in the present study. However, in patients who were converted to mastectomy that involved removing all subcutaneous breast tissue [5], Pecs 1 block as injection between the pectoralis major and minor would be necessary because pectoralis muscle was exposed. [27].

In fact, most of the patients in the present study were able to experience hypoesthesia in the skin segment, which fully covered the incision site. However, the majority of patients reported persistent chest pain after nerve block despite this sensory depression. This finding is probably the result of the role of other pain pathways such as the pectoral nerve, in addition to branches of the intercostal nerve, in pain after lumpectomy.

SIFB is thought to be advantageous in two theoretical aspects. First, modified Pecs 2 block results in greater diffusion within the serratus-intercostal plane caudally because the injection is located within a poorly distensible small neurovascular space, and respiratory movements allow local anesthetic spread through the space. Second, SIFB can avoid unnecessary long thoracic nerve blocks located above the SAM $[22,28]$.

The literature describes two needle approaches in SIFB. The anterior approach involves approaching below the lateral one-third of the clavicle. After identification of the pectoral muscles, the thoraco-acromial artery, the cephalic vein, the SAM resting on the ribs, and the EIM between the ribs, the needle is introduced in plane in the medial to lateral direction, and the needle tip is placed between the SAM and the
EIM. The injection is performed at the level of the second rib, below the SAM $[22,23]$.

In the lateral approach, the probe is placed in the mid-axillary line at the level of the 6th intercostal space. After identifying the SAM, intercostal muscles, ribs, pleura, and lung, the block needle is inserted until the tip is between the SAM and the EIM from caudal to cranial with the in-plane technique $[13,20]$. Because we adopted the anterior approach in the present study, it was not necessary to change the posture for the nerve block after surgery Therefore it was easy to perform PIFB afterward, because the investigator was positioned at the head of the patient. In addition, the inserted needle was targeted to the second rib, and the local anesthetic was injected with the tip of the needle in direct contact with the ribs and at the same time confirming the detachment of the muscle layer. Accordingly, it is easier to inject local anesthetics between the muscle layers than when the needle is targeted to the muscles, and complications such as pneumothorax can be avoided. With this procedure, more complete blockage of the intercostobrachial nerve and the branch of the second intercostal nerve is expected. It is thought that targeting the second rib also has the advantage of controlling axillary pain.

There is a difficulty in controlling postoperative pain in female patients. Sex is a risk factor for PONV in women. The use of opioid analgesics is usually required in patients undergoing surgery owing to moderate to severe pain, which is associated with a high risk of PONV [6]. Although lumpectomy is a minor surgery compared with other breast surgeries [5], postoperative pain after lumpectomy is not negligible, and it can increase the use of opioid analgesics, delay recovery after surgery, and lead to a chronic pain state such as postmastectomy pain syndrome [1-4,29]. Therefore, strategies for female patients to reduce PONV and control pain after surgery should be actively used. In this context, the effects of PIFB and SIFB shown in the current study are noteworthy.

The present study has several limitations. First, although the NRS pain score was higher in the control group than in the block group during patients' stay in the PACU, there was no difference in the NRS pain score between the two groups after more than 3 hours postoperatively. The reason for this finding seems to be that a greater amount of intravenous opioid analgesics was administered in the control group. Second, SIFB and PIFB may be insufficient blocks if the lumpectomy site is an upper quadrant innervated by the supracla- 
vicular nerve.

In conclusion, although lumpectomy is a less invasive procedure than other breast surgeries [5], thoracic interfascial plane block (SIFB and PIFB) is effective for multimodal analgesia after breast lumpectomy because it reduces opioid usage and increases patient satisfaction compared to the use of intravenous PCA alone.

\section{CONFLICTS OF INTEREST}

No potential conflict of interest relevant to this article was reported.

\section{ORCID}

Yeojung Kim: https://orcid.org/0000-0002-2079-670X

Chahyun Oh: https://orcid.org/0000-0001-8344-4245

Sookyoung Youn: https://orcid.org/0000-0002-8461-0599

Sangwon Yun: https://orcid.org/0000-0001-8233-4167

Hyunwoo Park: https://orcid.org/0000-0002-1113-4181

Wonhyung Lee: https://orcid.org/0000-0002-1964-7141

Yoon-Hee Kim: https://orcid.org/0000-0002-8282-610X

YoungKwon Ko: https://orcid.org/0000-0002-0178-6346

\section{REFERENCES}

1. Andersen KG, Kehlet H. Persistent pain after breast cancer treatment: a critical review of risk factors and strategies for prevention. J Pain 2011; 12: 725-46.

2. Fassoulaki A, Melemeni A, Staikou C, Triga A, Sarantopoulos C. Acute postoperative pain predicts chronic pain and long-term analgesic requirements after breast surgery for cancer. Acta Anaesthesiol Belg 2008; 59: 241-8.

3. Iohom G, Abdalla H, O’Brien J, Szarvas S, Larney V, Buckley E, et al. The associations between severity of early postoperative pain, chronic postsurgical pain and plasma concentration of stable nitric oxide products after breast surgery. Anesth Analg 2006; 103: 995-1000.

4. Katz J, Jackson M, Kavanagh BP, Sandler AN. Acute pain after thoracic surgery predicts long-term post-thoracotomy pain. Clin J Pain 1996; 12: 50-5.

5. Woodworth GE, Ivie RMJ, Nelson SM, Walker CM, Maniker RB. Perioperative breast analgesia: a qualitative review of anatomy and regional techniques. Reg Anesth Pain Med 2017; 42: 609-31.

6. Apfel CC, Läärä E, Koivuranta M, Greim CA, Roewer N. A simpli- fied risk score for predicting postoperative nausea and vomiting: conclusions from cross-validations between two centers. Anesthesiology 1999; 91: 693-700.

7. Lynch EP, Welch KJ, Carabuena JM, Eberlein TJ. Thoracic epidural anesthesia improves outcome after breast surgery. Ann Surg 1995; 222: 663-9.

8. Naja MZ, Ziade MF, Lönnqvist PA. Nerve-stimulator guided paravertebral blockade vs. general anaesthesia for breast surgery: a prospective randomized trial. Eur J Anaesthesiol 2003; 20: 897-903.

9. Klein SM, Bergh A, Steele SM, Georgiade GS, Greengrass RA. Thoracic paravertebral block for breast surgery. Anesth Analg 2000; 90: 1402-5.

10. Moller JF, Nikolajsen L, Rodt SA, Ronning H, Carlsson PS. Thoracic paravertebral block for breast cancer surgery: a randomized double-blind study. Anesth Analg 2007; 105: 1848-51.

11. Blanco R, Fajardo M, Parras Maldonado T. Ultrasound description of Pecs II (modified Pecs I): a novel approach to breast surgery. Rev Esp Anestesiol Reanim 2012; 59: 470-5.

12. Blanco R. The 'pecs block': a novel technique for providing analgesia after breast surgery. Anaesthesia 2011; 66: 847-8.

13. de la Torre PA, García PD, Alvarez SL, Miguel FJ, Pérez MF. A novel ultrasound-guided block: a promising alternative for breast analgesia. Aesthet Surg J 2014; 34: 198-200.

14. Fajardo-Pérez M, Altınpulluk EY, García-Miguel J, QuintanaGordon B. Ultrasound-guided continuous interpectoral block for patient undergoing mastectomy and axillary clearance. Turk J Anaesthesiol Reanim 2017; 45: 112-5.

15. Bashandy GM, Abbas DN. Pectoral nerves I and II blocks in multimodal analgesia for breast cancer surgery: a randomized clinical trial. Reg Anesth Pain Med 2015; 40: 68-74.

16. M N, Pandey RK, Sharma A, Darlong V, Punj J, Sinha R, et al. Pectoral nerve blocks to improve analgesia after breast cancer surgery: a prospective, randomized and controlled trial. J Clin Anesth 2018; 45: 12-7.

17. Versyck B, van Geffen GJ, Van Houwe P. Prospective double blind randomized placebo-controlled clinical trial of the pectoral nerves (Pecs) block type II. J Clin Anesth 2017; 40: 46-50.

18. Liu V, Mariano ER, Prabhakar C. Pecto-intercostal fascial block for acute poststernotomy pain: a case report. A A Pract 2018; 10: 319-22.

19. Hong B, Yoon SH, Youn AM, Kim BJ, Song S, Yoon Y. Thoracic interfascial nerve block for breast surgery in a pregnant woman: a case report. Korean J Anesthesiol 2017; 70: 209-12.

20. Blanco R, Parras T, McDonnell JG, Prats-Galino A. Serratus plane block: a novel ultrasound-guided thoracic wall nerve block. Anaesthesia 2013; 68: 1107-13. 
21. Ohgoshi Y, Yokozuka M, Terajima K. [Serratus-intercostal plane block for brest surgery]. Masui 2015; 64: 610-4. Japanese.

22. Pérez MF, Duany O, de la Torre PA. Redefining PECS blocks for postmastectomy analgesia. Reg Anesth Pain Med 2015; 40: 72930.

23. Pérez MF, Miguel JG, de la Torre PA. A new approach to pectoralis block. Anaesthesia 2013; 68: 430.

24. Leiman D, Barlow M, Carpin K, Piña EM, Casso D. Medial and lateral pectoral nerve block with liposomal bupivacaine for the management of postsurgical pain after submuscular breast augmentation. Plast Reconstr Surg Glob Open 2015; 2: e282.

25. Jaspars JJ, Posma AN, van Immerseel AA, Gittenberger-de Groot AC. The cutaneous innervation of the female breast and nippleareola complex: implications for surgery. Br J Plast Surg 1997; 50: 249-59.
26. Kulhari S, Bharti N, Bala I, Arora S, Singh G. Efficacy of pectoral nerve block versus thoracic paravertebral block for postoperative analgesia after radical mastectomy: a randomized controlled trial. Br J Anaesth 2016; 117: 382-6.

27. Hetta DF, Rezk KM. Pectoralis-serratus interfascial plane block vs thoracic paravertebral block for unilateral radical mastectomy with axillary evacuation. J Clin Anesth 2016; 34: 91-7.

28. Torre PA, Jones JW Jr, Álvarez SL, Garcia PD, Miguel FJG, Rubio EMM, et al. [Axillary local anesthetic spread after the thoracic interfacial ultrasound block - a cadaveric and radiological evaluation]. Rev Bras Anestesiol 2017; 67: 555-64. Portuguese.

29. Exadaktylos AK, Buggy DJ, Moriarty DC, Mascha E, Sessler DI. Can anesthetic technique for primary breast cancer surgery affect recurrence or metastasis? Anesthesiology 2006; 105: 660-4. 